\title{
On the Normed Space of Equivalence Classes of Fuzzy Numbers
}

\author{
Dong Qiu, Chongxia Lu, and Wei Zhang \\ College of Mathematics and Physics, Chongqing University of Posts and Telecommunications, Nanan, Chongqing 400065, China
}

Correspondence should be addressed to Dong Qiu; dongqiumath@163.com

Received 9 June 2013; Accepted 30 July 2013

Academic Editors: T. Allahviranloo and A. N. Yannacopoulos

Copyright (C) 2013 Dong Qiu et al. This is an open access article distributed under the Creative Commons Attribution License, which permits unrestricted use, distribution, and reproduction in any medium, provided the original work is properly cited.

We study the norm induced by the supremum metric on the space of fuzzy numbers. And then we propose a method for constructing a norm on the quotient space of fuzzy numbers. This norm is very natural and works well with the induced metric on the quotient space.

\section{Introduction}

Since the concept of fuzzy numbers was firstly introduced in the 1970s, it has been studied extensively from many different aspects of the theory and applications such as fuzzy topology, fuzzy analysis, fuzzy logic, and fuzzy decision making (see, e.g., [1-6]). The operations in the set of fuzzy numbers are usually obtained by the Zadeh extension principle [7-9]. In the study of algebraic structures and topological structures for fuzzy numbers, many results have been obtained (see, e.g., $[10-19])$.

In the classical mathematics, if $X$ is a normed space with norm $\|\cdot\|$, it is readily checked that the formula $d(x, y)=$ $\|x-y\|$, for $x, y \in X$, defines a metric $d$ on $X$. Thus a normed space is naturally a metric space and all metric space concepts are meaningful. However, we will show that such proposition does not hold true for the well known supremum metric on the space of fuzzy numbers. To overcome this weakness, we will consider the quotient space of fuzzy numbers up to an equivalence relation which is introduced by Mareš $[20,21]$ and is studied extensively by many researchers [4, 12, 22-24]. We will propose a method for constructing a norm on the quotient space of fuzzy numbers. This norm is very natural and works well with the induced metric on the quotient space.

\section{Preliminaries}

A fuzzy set of $\mathbb{R}$ is a function $\mu: \mathbb{R} \rightarrow[0,1]$. For each such fuzzy set $\mu$, we denote by $[\mu]^{a}=\{x \in \mathbb{R}: \mu(x) \geq a\}$ for any $a \in(0,1]$ its $a$-level set. We define the set $[\mu]^{0}$ by $[\mu]^{0}=\overline{\{x \in \mathbb{R}: \mu(x)>0\}}$, where $\bar{A}$ denotes the closure of a set $A$. A fuzzy number $\mu$ is a fuzzy set with nonempty bounded closed level sets $[\mu]^{a}=\left[\mu_{L}(a), \mu_{R}(a)\right]$ for all $a \epsilon$ $[0,1]$. We denote the class of fuzzy numbers by $\mathscr{F}$. Notice that the real numbers $\mathbb{R}$ can be imbedded in $\mathscr{F}$ by defining a fuzzy number as follows

$$
\mathbf{a}(x)= \begin{cases}1, & \text { if } x=a, \\ 0, & \text { otherwise }\end{cases}
$$

for each $a \in \mathbb{R}$.

For any $\mu, \nu \in \mathscr{F}$ and $a \in \mathbb{R}$, owing to Zadeh's extension principle [7-9], scalar multiplication and addition are defined for any $x \in \mathbb{R}$ by

$$
\begin{aligned}
& (a \times \mu)(x)=(a \mu)(x)= \begin{cases}\mu\left(\frac{x}{a}\right), & \text { if } a \neq 0, \\
0, & \text { if } a=0,\end{cases} \\
& (\mu+\nu)(x)=\sup _{x_{1}, x_{2}: x_{1}+x_{2}=x} \min \left\{\mu\left(x_{1}\right), \nu\left(x_{2}\right)\right\} .
\end{aligned}
$$

For any $\mu \in \mathscr{F}$, we define the fuzzy number $-\mu \in \mathscr{F}$ by $-\mu=(-1) \times \mu$, that is, $-\mu(x)=\mu(-x)$, for all $x \in \mathbb{R}$. Since the addition does not possess an inverse subtraction, $\mathscr{F}$ is not a real vector space. We say that a fuzzy number $s \in \mathscr{F}$ is symmetric [20], if

$$
s(x)=s(-x),
$$

for all $x \in \mathbb{R}$, that is, $s=-s$. The set of all symmetric fuzzy numbers will be denoted by $\mathcal{S}$. 
Definition 1 (see [22]). Let $\mu, \nu \in \mathscr{F}$. We say that $\mu$ is equivalent to $\nu$ and write $\mu \sim \nu$ if and only if there exist symmetric fuzzy numbers $s_{1}, s_{2} \in \mathcal{S}$ such that

$$
\mu+s_{1}=v+s_{2}
$$

The equivalence relation defined above is reflexive, symmetric, and transitive [20]. Let $\langle\mu\rangle$ denote the equivalence class containing the element $\mu$ and denote the set of equivalence classes by $\mathscr{F} / \mathcal{S}$. By the level set representations for fuzzy numbers, one can easily prove the following lemmas.

Lemma 2 (see [25]). For any $\mu \in \mathscr{F}, \mu-\mu \in \mathcal{S}$.

Lemma 3 (see [25]). For any $\mu, v \in \mathscr{F}, \mu \sim \nu$ if and only if $\mu-\nu \in \mathcal{S}$.

Definition 4 (see [26]). For a fuzzy number $\mu$, we define a function $\mu_{M}:[0,1] \rightarrow \mathbb{R}$ by assigning the midpoint of each $a$-level set to $\mu_{M}(a)$ for all $a \in[0,1]$, that is,

$$
\mu_{M}(a)=\frac{\mu_{L}(a)+\mu_{R}(a)}{2}
$$

Lemma 5 (see [25]). For any $\mu, v \in \mathscr{F}, \mu \sim \nu$ if and only if $\mu_{M}=v_{M}$.

Lemma 6 (see $[26]) .(\mathscr{F} / \mathcal{S},+, \times)$ is a real vector space.

\section{Main Results}

In this section, we will give a norm structure on $\mathscr{F} / \mathcal{S}$ which is compatible with a metric. For the family of fuzzy numbers $\mathscr{F}$, the $d_{\infty}$-metric is induced by the Hausdorff metric as [17]

$$
\begin{aligned}
d_{\infty}(\mu, \nu) & =\sup _{0 \leq a \leq 1} H\left([\mu]^{a},[\nu]^{a}\right) \\
& =\sup _{0 \leq a \leq 1} \max \left\{\left|\mu_{L}(a)-\nu_{L}(a)\right|,\left|\mu_{R}(a)-v_{R}(a)\right|\right\}
\end{aligned}
$$

for all $\mu, v \in \mathscr{F}$.

Define a function $\|\cdot\|: \mathscr{F} \rightarrow \mathbb{R}$ as

$$
\|\mu\|=d(\mu, \mathbf{0})=\sup _{0 \leq a \leq 1} \max \left\{\left|\mu_{L}(a)\right|,\left|\mu_{R}(a)\right|\right\} .
$$

Theorem 7. The function $\|\cdot\|$ in (7) is a norm on $\mathscr{F}$.

Proof. (i) It is obvious that $\|\mu\| \geq 0$ for all $\mu \in \mathscr{F}$ and $\|\mu\|=0$ if $\mu=\mathbf{0}$. Conversely, if $\mu \neq \mathbf{0}$, then we have that there exists $a_{0} \in[0,1]$ such that $\left[\mu_{L}\left(a_{0}\right), \mu_{R}\left(a_{0}\right)\right] \neq\{0\}$. Thus we have $\|\mu\|>$ 0 .

(ii) For all $\mu \in \mathscr{F}$ and $b \in \mathbb{R}$, we have

$$
\begin{aligned}
\|b \mu\| & =\sup _{0 \leq a \leq 1} \max \left\{|b|\left|\mu_{L}(a)\right|,|b|\left|\mu_{R}(a)\right|\right\} \\
& =|b|\|\mu\| .
\end{aligned}
$$

(iii) For all $\mu, v \in \mathscr{F}$, we have that

$$
\begin{aligned}
\|\mu+\nu\|= & \sup _{0 \leq a \leq 1} \max \left\{\left|\mu_{L}(a)+\nu_{L}(a)\right|,\left|\mu_{R}(a)+v_{R}(a)\right|\right\} \\
\leq & \sup _{0 \leq a \leq 1} \max \left\{\left|\mu_{L}(a)\right|+\left|\nu_{L}(a)\right|,\left|\mu_{R}(a)\right|+\left|\nu_{R}(a)\right|\right\} \\
\leq & \sup _{0 \leq a \leq 1} \max \left\{\left|\mu_{L}(a)\right|,\left|\mu_{R}(a)\right|\right\} \\
& +\sup _{0 \leq a \leq 1} \max \left\{\left|\nu_{L}(a)\right|,\left|\nu_{R}(a)\right|\right\} \\
= & \|\mu\|+\|\nu\| .
\end{aligned}
$$

We conclude that $\|\cdot\|$ is a norm on $\mathscr{F}$.

Although $\|\cdot\|$ is a norm on $\mathscr{F}$, the function $d: \mathscr{F} \times \mathscr{F} \rightarrow$ $\mathbb{R}$ induced by $\|\cdot\|$ as $d(\mu, \nu)=\|\mu-\nu\|$ is not a metric on $\mathscr{F}$. Consequently, we get that $d_{\infty}(\mu, v) \neq\|\mu-\nu\|$.

Theorem 8. The function d has the following properties:

(i) $d(\mu, v) \geq 0$, for any $\mu, v \in \mathscr{F}$;

(ii) $d(\mu, \nu)=d(\nu, \mu)$, for any $\mu, \nu \in \mathscr{F}$;

(iii) $d(\mu, \nu) \leq d(\mu, \omega)+d(\omega, \nu)$, for any $\mu, \nu, \omega \in \mathscr{F}$;

(iv) $d(\mu, \mu) \neq 0$, for any fuzzy number $\mu \notin \mathbb{R}$.

Proof. (i) By the definition of $d$, it is obvious that $d(\mu, \nu) \geq 0$, for any $\mu, \nu \in \mathscr{F}$.

(ii) For all $\mu, \nu \in \mathscr{F}$, we have that

$$
\begin{aligned}
d(\mu, \nu) & =\|\mu-v\| \\
& =\sup _{0 \leq a \leq 1} \max \left\{\left|\mu_{L}(a)-v_{R}(a)\right|,\left|\mu_{R}(a)-\nu_{L}(a)\right|\right\} \\
& =\sup _{0 \leq a \leq 1} \max \left\{\left|\nu_{L}(a)-\mu_{R}(a)\right|,\left|\nu_{R}(a)-\mu_{L}(a)\right|\right\} \\
& =\|\nu-\mu\| \\
& =d(\nu, \mu) .
\end{aligned}
$$

(iii) In order to prove the triangle inequality, for any fixed $a \in[0,1]$, and for any $\mu, \nu, \omega \in \mathscr{F}$, we only proof the following six cases. Similarly, the others can be proved.

Case $1\left(\mu_{L}(a) \leq \nu_{L}(a) \leq \mu_{R}(a) \leq \nu_{R}(a)\right.$ and $\left.\omega_{L}(a) \leq \mu_{L}(a)\right)$. In this case we have that

$$
\begin{aligned}
\max & \left\{\left|\mu_{L}(a)-v_{R}(a)\right|,\left|\mu_{R}(a)-v_{L}(a)\right|\right\} \\
= & \left|\mu_{L}(a)-v_{R}(a)\right| \\
\leq & \left|\omega_{L}(a)-v_{R}(a)\right| \\
\leq & \max \left\{\left|\mu_{L}(a)-\omega_{R}(a)\right|,\left|\mu_{R}(a)-\omega_{L}(a)\right|\right\} \\
& +\max \left\{\left|\omega_{L}(a)-v_{R}(a)\right|,\left|\omega_{R}(a)-v_{L}(a)\right|\right\} .
\end{aligned}
$$


Case $2\left(\mu_{L}(a) \leq \nu_{L}(a) \leq \mu_{R}(a) \leq \nu_{R}(a)\right.$ and $\mu_{L}(a) \leq \omega_{L}(a) \leq$ $\left.\omega_{R}(a) \leq v_{R}(a)\right)$. In this case we have that

$$
\begin{aligned}
\max & \left\{\left|\mu_{L}(a)-v_{R}(a)\right|,\left|\mu_{R}(a)-v_{L}(a)\right|\right\} \\
= & \left|\mu_{L}(a)-v_{R}(a)\right| \\
\leq & \left|\mu_{L}(a)-\omega_{R}(a)\right|+\left|\omega_{L}(a)-v_{R}(a)\right| \\
\leq & \max \left\{\left|\mu_{L}(a)-\omega_{R}(a)\right|,\left|\mu_{R}(a)-\omega_{L}(a)\right|\right\} \\
& \quad+\max \left\{\left|\omega_{L}(a)-v_{R}(a)\right|,\left|\omega_{R}(a)-v_{L}(a)\right|\right\} .
\end{aligned}
$$

Case $3\left(\mu_{L}(a) \leq \nu_{L}(a) \leq \nu_{R}(a) \leq \mu_{R}(a)\right.$ and $\omega_{L}(a) \leq$ $\left.\mu_{L}(a)\right)$. In this case we have that

$$
\begin{aligned}
\max & \left\{\left|\mu_{L}(a)-v_{R}(a)\right|,\left|\mu_{R}(a)-v_{L}(a)\right|\right\} \\
\leq & \left|\mu_{L}(a)-v_{R}(a)\right| \\
\leq & \left|\omega_{L}(a)-\mu_{R}(a)\right| \\
\leq & \max \left\{\left|\mu_{L}(a)-\omega_{R}(a)\right|,\left|\mu_{R}(a)-\omega_{L}(a)\right|\right\} \\
& \quad+\max \left\{\left|\omega_{L}(a)-v_{R}(a)\right|,\left|\omega_{R}(a)-v_{L}(a)\right|\right\} .
\end{aligned}
$$

Case $4\left(\mu_{L}(a) \leq \omega_{L}(a) \leq \omega_{R}(a) \leq v_{L}(a) \leq v_{R}(a) \leq \mu_{R}(a)\right)$. If $\max \left\{\left|\mu_{L}(a)-v_{R}(a)\right|,\left|\mu_{R}(a)-v_{L}(a)\right|\right\}=\left|\mu_{L}(a)-v_{R}(a)\right|$, then we have that

$$
\begin{aligned}
\max & \left\{\left|\mu_{L}(a)-\nu_{R}(a)\right|,\left|\mu_{R}(a)-\nu_{L}(a)\right|\right\} \\
= & \left|\mu_{L}(a)-v_{R}(a)\right| \\
= & \left|\mu_{L}(a)-\omega_{L}(a)\right|+\left|\omega_{L}(a)-\nu_{R}(a)\right| \\
\leq & \left|\mu_{L}(a)-\omega_{R}(a)\right|+\left|\omega_{L}(a)-v_{R}(a)\right| \\
\leq & \max \left\{\left|\mu_{L}(a)-\omega_{R}(a)\right|,\left|\mu_{R}(a)-\omega_{L}(a)\right|\right\} \\
& +\max \left\{\left|\omega_{L}(a)-v_{R}(a)\right|,\left|\omega_{R}(a)-\nu_{L}(a)\right|\right\} .
\end{aligned}
$$

Otherwise, we have that

$$
\begin{aligned}
\max & \left\{\left|\mu_{L}(a)-v_{R}(a)\right|,\left|\mu_{R}(a)-v_{L}(a)\right|\right\} \\
= & \left|\mu_{R}(a)-v_{L}(a)\right| \\
\leq & \left|\mu_{R}(a)-\omega_{L}(a)\right| \\
\leq & \max \left\{\left|\mu_{L}(a)-\omega_{R}(a)\right|,\left|\mu_{R}(a)-\omega_{L}(a)\right|\right\} \\
& \quad+\max \left\{\left|\omega_{L}(a)-v_{R}(a)\right|,\left|\omega_{R}(a)-v_{L}(a)\right|\right\} .
\end{aligned}
$$

Case $5\left(\mu_{L}(a) \leq v_{L}(a) \leq \omega_{L}(a) \leq \omega_{R}(a) \leq v_{R}(a) \leq \mu_{R}(a)\right)$. In this case we have that

$$
\begin{aligned}
& \left|\mu_{L}(a)-\nu_{R}(a)\right| \leq\left|\mu_{L}(a)-\omega_{R}(a)\right|+\left|\omega_{L}(a)-\nu_{R}(a)\right| \\
& \left|\mu_{R}(a)-\nu_{L}(a)\right| \leq\left|\omega_{L}(a)-\nu_{R}(a)\right|+\left|\omega_{R}(a)-\nu_{L}(a)\right| .
\end{aligned}
$$

Consequently, we have that

$$
\begin{aligned}
\max & \left\{\left|\mu_{L}(a)-\nu_{R}(a)\right|,\left|\mu_{R}(a)-\nu_{L}(a)\right|\right\} \\
\leq & \max \left\{\left|\mu_{L}(a)-\omega_{R}(a)\right|,\left|\mu_{R}(a)-\omega_{L}(a)\right|\right\} \\
& +\max \left\{\left|\omega_{L}(a)-\nu_{R}(a)\right|,\left|\omega_{R}(a)-\nu_{L}(a)\right|\right\} .
\end{aligned}
$$

Case $6\left(\mu_{L}(a) \leq \omega_{L}(a) \leq \nu_{L}(a) \leq \nu_{R}(a) \leq \omega_{R}(a) \leq \mu_{R}(a)\right)$. In this case we have that

$$
\begin{aligned}
& \left|\mu_{L}(a)-\nu_{R}(a)\right| \leq\left|\mu_{L}(a)-\omega_{R}(a)\right|, \\
& \left|\mu_{R}(a)-\nu_{L}(a)\right| \leq\left|\mu_{R}(a)-\omega_{L}(a)\right| .
\end{aligned}
$$

Consequently, we have that

$$
\begin{aligned}
\max & \left\{\left|\mu_{L}(a)-\nu_{R}(a)\right|,\left|\mu_{R}(a)-\nu_{L}(a)\right|\right\} \\
\leq & \max \left\{\left|\mu_{L}(a)-\omega_{R}(a)\right|,\left|\mu_{R}(a)-\omega_{L}(a)\right|\right\} \\
& +\max \left\{\left|\omega_{L}(a)-v_{R}(a)\right|,\left|\omega_{R}(a)-v_{L}(a)\right|\right\} .
\end{aligned}
$$

From what is proved above, we can get that

$$
\begin{aligned}
d(\mu, v)= & \|\mu-v\| \\
= & \sup _{0 \leq a \leq 1} \max \left\{\left|\mu_{L}(a)-v_{R}(a)\right|,\left|\mu_{R}(a)-v_{L}(a)\right|\right\} \\
\leq & \sup _{0 \leq a \leq 1} \max \left\{\left|\mu_{L}(a)-\omega_{R}(a)\right|,\left|\mu_{R}(a)-\omega_{L}(a)\right|\right\} \\
& +\sup _{0 \leq a \leq 1} \max \left\{\left|\omega_{L}(a)-v_{R}(a)\right|,\left|\omega_{R}(a)-v_{L}(a)\right|\right\} \\
= & \|\mu-\omega\|+\|\omega-\nu\| \\
= & d(\mu, \omega)+d(\omega, \nu) .
\end{aligned}
$$

(iv) Since $\mu \notin \mathbb{R}$, there exists $a_{0} \in[0,1]$ such that $\mu_{R}\left(a_{0}\right)-$ $\mu_{L}\left(a_{0}\right)>0$. Thus we have that

$$
\begin{aligned}
d(\mu, \mu) & =\|\mu-\mu\| \\
& =\sup _{0 \leq a \leq 1}\left|\mu_{L}(a)-\mu_{R}(a)\right| \\
& >\mu_{R}\left(a_{0}\right)-\mu_{L}\left(a_{0}\right) \\
& >0 .
\end{aligned}
$$

In order to induce a metric which is compatible with the norm, we consider the quotient space of fuzzy numbers. It is very natural to define a function $\|\cdot\|: \mathscr{F} / \mathcal{S} \rightarrow \mathbb{R}$ as

$$
\|\langle\mu\rangle\|=\inf _{\nu \in\langle\mu\rangle}\|\nu\|,
$$

for each $\langle\mu\rangle \in \mathscr{F} / \mathcal{S}$. 
Theorem 9. The function $\|\cdot\|$ in (22) is a norm on $\mathscr{F} / \mathcal{S}$.

Proof. (i) It is obvious that $\|\langle\mu\rangle\| \geq 0$ for all $\mu \in \mathscr{F}$ and $\|\langle\mu\rangle\|=0$ if $\langle\mu\rangle=\langle\mathbf{0}\rangle$. Conversely, if $\langle\mu\rangle \neq\langle\mathbf{0}\rangle$, from Lemma 5, it follows that the midpoint function $\mu_{M} \neq \mathbf{0}_{M}$. Thus we have

$$
\begin{aligned}
\|\langle\mu\rangle\| & =\inf _{\nu \in\langle\mu\rangle}\|\nu\| \\
& =\inf _{\nu \in\langle\mu\rangle}\left(\sup _{0 \leq a \leq 1} \max \left\{\left|\nu_{L}(a)\right|,\left|\nu_{R}(a)\right|\right\}\right) \\
& \geq \inf _{\nu \in\langle\mu\rangle}\left(\sup _{0 \leq a \leq 1} \frac{\left|\nu_{L}(a)+\nu_{R}(a)\right|}{2}\right) \\
& =\sup _{0 \leq a \leq 1}\left|\mu_{M}(a)\right| \\
& >0 .
\end{aligned}
$$

(ii) For all $\langle\mu\rangle \in \mathscr{F} / \mathcal{S}$ and $b \in \mathbb{R}$, we have

$$
\begin{aligned}
\|b\langle\mu\rangle\| & =\|\langle b \mu\rangle\| \\
& =\inf _{\nu \in\langle b \mu\rangle}\|v\| \\
& =\inf _{b \nu^{\prime} \in\langle b \mu\rangle}\left\|b \nu^{\prime}\right\| \\
& =\inf _{v^{\prime} \in\langle\mu\rangle}\left\|b \nu^{\prime}\right\| \\
& =|b|\|\langle\mu\rangle\| .
\end{aligned}
$$

(iii) For all $\langle\mu\rangle,\langle\nu\rangle \in \mathscr{F} / \mathcal{S}$, we have that

$$
\begin{aligned}
\|\langle\mu\rangle+\langle\nu\rangle\| & =\|\langle\mu+\nu\rangle\| \\
& =\inf _{\omega \in\langle\mu+\nu\rangle}\|\omega\| \\
& \leq \inf _{\mu^{\prime} \in\langle\mu\rangle, \nu^{\prime} \in\langle\nu\rangle}\|\mu+\nu\| \\
& \leq \inf _{\mu^{\prime} \in\langle\mu\rangle, \nu^{\prime} \in\langle\nu\rangle}(\|\mu\|+\|\nu\|) \\
& \leq \inf _{\mu^{\prime} \in\langle\mu\rangle}\|\mu\|+\inf _{\nu^{\prime} \in\langle\nu\rangle}\|\nu\| \\
& =\|\langle\mu\rangle\|+\|\langle\nu\rangle\| .
\end{aligned}
$$

We conclude that $\|\cdot\|$ is a norm on $\mathscr{F} / \mathcal{S}$.

Now we show that the function $\rho: \mathscr{F} / \mathcal{S} \times \mathscr{F} / \mathcal{S} \rightarrow \mathbb{R}$ induced by $\|\cdot\|$ as $\rho(\langle\mu\rangle,\langle\nu\rangle)=\|\langle\mu-\nu\rangle\|$ is exactly a metric on $\mathscr{F} / \mathcal{S}$.

Theorem 10. The function $\rho$ is a metric on $\mathscr{F} / \mathcal{S}$.

Proof. (i) By the definition of $\rho$, it is obvious that $\rho(\langle\mu\rangle,\langle\nu\rangle) \geq 0$, for any $\langle\mu\rangle,\langle\nu\rangle \in \mathscr{F} / \mathcal{S}$. If $\rho(\langle\mu\rangle,\langle\nu\rangle)=0$, then $\|\langle\mu-\nu\rangle\|=0$. Thus by Theorem 9, we get $\langle\mu\rangle=\langle\nu\rangle$. In addition, by Lemma 2 we have $\rho(\langle\mu\rangle,\langle\mu\rangle)=\|\langle\mu-\mu\rangle\|=0$.

(ii) For all $\langle\mu\rangle,\langle\nu\rangle \in \mathscr{F} / \mathcal{S}$, we have that

$$
\begin{aligned}
\rho(\langle\mu\rangle,\langle\nu\rangle) & =\|\langle\mu-\nu\rangle\|=\|(-1)\langle\nu-\mu\rangle\| \\
& =\|\nu-\mu\|=\rho(\langle\nu\rangle,\langle\mu\rangle) .
\end{aligned}
$$

(iii) For any $\langle\mu\rangle,\langle\nu\rangle,\langle\omega\rangle \in \mathscr{F} / \mathcal{S}$, we have

$$
\begin{aligned}
\rho(\langle\mu\rangle,\langle\nu\rangle) & =\|\langle\mu-\nu\rangle\| \\
& =\|\langle\mu-\omega+\omega-\nu\rangle\| \\
& \leq\|\langle\mu-\omega\rangle\|+\|\langle\omega-\nu\rangle\| \\
& =\rho(\langle\mu\rangle,\langle\omega\rangle)+\rho(\langle\omega\rangle,\langle\nu\rangle) .
\end{aligned}
$$

We conclude that $\rho$ is a metric on $\mathscr{F} / \mathcal{S}$.

\section{Conclusions}

In this present investigation, we studied the norm induced by the supremum metric $d_{\infty}$ on the space of fuzzy numbers. And then we proposed a method for constructing a norm on the quotient space of fuzzy numbers. This norm is very natural and works well with the induced metric on $\mathscr{F} / \mathcal{S}$. The works in this paper enable us to study the fuzzy numbers in the new environment. We hope that our results in this paper may lead to significant, new, and innovative results in other related fields.

\section{Acknowledgments}

This work was supported by the Mathematical Tianyuan Foundation of China (Grant no. 11126087), the National Natural Science Foundation of China (Grant no. 11201512) and the Natural Science Foundation Project of CQ CSTC (cstc2012jjA00001).

\section{References}

[1] B. Bede and S. G. Gal, "Generalizations of the differentiability of fuzzy-number-valued functions with applications to fuzzy differential equations," Fuzzy Sets and Systems, vol. 151, no. 3, pp. 581-599, 2005.

[2] D. Dubois and H. Prade, "Operations on fuzzy numbers," International Journal of Systems Science, vol. 9, no. 6, pp. 613-626, 1978.

[3] D. Dubois and H. Prade, Fuzzy Sets and Systems, Academic Press, New York, NY, USA, 1980.

[4] D. H. Hong, "An additive decomposition of fuzzy numbers," Kybernetika, vol. 39, no. 3, pp. 289-294, 2003.

[5] M. Michta, "On set-valued stochastic integrals and fuzzy stochastic equations," Fuzzy Sets and Systems, vol. 177, no. 1, pp. 119, 2011.

[6] L. Stefanini, "A generalization of Hukuhara difference and division for interval and fuzzy arithmetic," Fuzzy Sets and Systems, vol. 161, no. 11, pp. 1564-1584, 2010. 
[7] L. A. Zadeh, "The concept of alinguistic variable and its applications to approximate reasoning-I," Information Sciences, vol. 8, no. 3, pp. 199-249, 1975.

[8] L. A. Zadeh, "The concept of alinguistic variable and its applications to approximate reasoning,-II," Information Sciences, vol. 8, no. 4, pp. 301-357, 1975.

[9] L. A. Zadeh, "The concept of a linguistic variable and its application to approximate reasoning-III," Information Sciences, vol. 9, no. 1, pp. 43-80, 1975.

[10] A. M. Bica, "Algebraic structures for fuzzy numbers from categorial point of view," Soft Computing, vol. 11, no. 11, pp. 10991105, 2007.

[11] D. Dubois and H. Prade, "Additions of interactive fuzzy numbers," IEEE Transactions on Automatic Control, vol. 26, no. 4, pp. 926-936, 1981.

[12] D. H. Hong and S. Lee, "Some algebraic properties and a distance measure for interval-valued fuzzy numbers," Information Sciences, vol. 148, no. 1-4, pp. 1-10, 2002.

[13] A. Maturo, "On some structures of fuzzy numbers," Iranian Journal of Fuzzy Systems, vol. 6, no. 4, pp. 49-59, 2009.

[14] R. Mesiar, "A note to the T-sum of L-R fuzzy numbers," Fuzzy Sets and Systems, vol. 79, no. 2, pp. 259-261, 1996.

[15] S. G. Pushkov, "Fuzzy modules with respect to a t-norm and some of their properties," Journal of Mathematical Sciences, vol. 154, no. 3, pp. 374-378, 2008.

[16] D. Qiu and L. Shu, "Notes on 'On the restudy of fuzzy complex analysis: part I and part II'” Fuzzy Sets and Systems, vol. 159, no. 16, pp. 2185-2189, 2008.

[17] D. Qiu and L. Shu, "Supremum metric on the space of fuzzy sets and common fixed point theorems for fuzzy mappings," Information Sciences, vol. 178, no. 18, pp. 3595-3604, 2008.

[18] D. Qiu, L. Shu, and Z. Mo, "Notes on fuzzy complex analysis," Fuzzy Sets and Systems, vol. 160, no. 11, pp. 1578-1589, 2009.

[19] D. Qiu, L. Shu, and Z. Mo, “On starshaped fuzzy sets," Fuzzy Sets and Systems, vol. 160, no. 11, pp. 1563-1577, 2009.

[20] M. Mareš, "Addition of fuzzy quantities: disjunctionconjunction approach," Kybernetika, vol. 25, no. 2, pp. 104-116, 1989.

[21] M. Mareš, "Additive decomposition of fuzzy quantities with finite supports," Fuzzy Sets and Systems, vol. 47, no. 3, pp. 341346, 1992.

[22] D. H. Hong and H. Y. Do, "Additive decomposition of fuzzy quantities," Information Sciences, vol. 88, no. 1-4, pp. 201-207, 1996.

[23] M. Mareš, "Algebraic equivalences over fuzzy quantities," Kybernetika, vol. 29, no. 2, pp. 122-132, 1993.

[24] M. Mareš, "Brief note on distributivity of triangular fuzzy numbers," Kybernetika, vol. 31, no. 5, pp. 451-457, 1995.

[25] D. Qiu and W. Zhang, "Symmetric fuzzy numbers and additive equivalence of fuzzy numbers," Soft Computing, vol. 17, no. 8, pp. 1471-1477, 2013.

[26] K. D. Jamison, "Possibilities as cumulative subjective probabilities and a norm on the space of congruence classes of fuzzy numbers motivated by an expected utility functional," Fuzzy Sets and Systems, vol. 111, no. 3, pp. 331-339, 2000. 


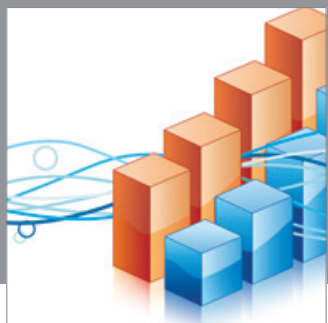

Advances in

Operations Research

mansans

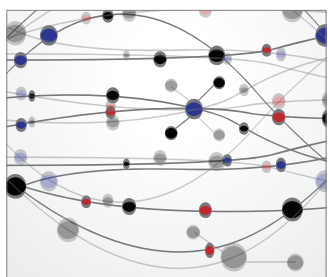

The Scientific World Journal
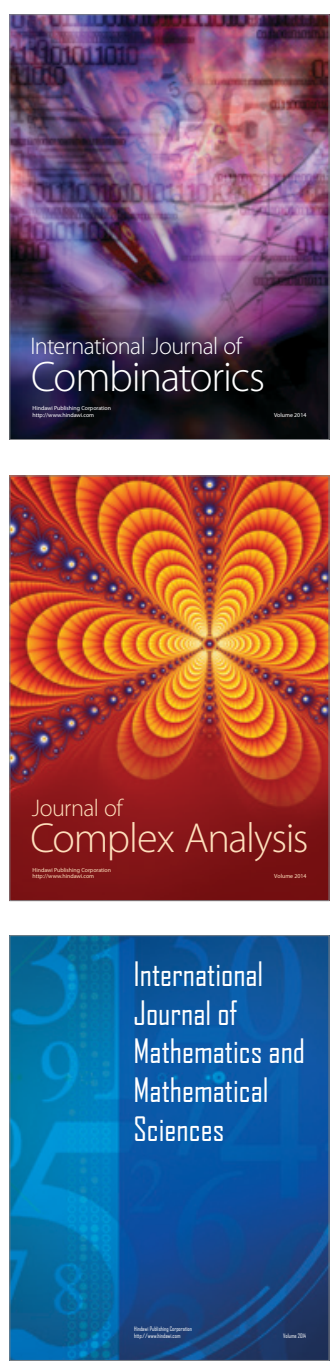
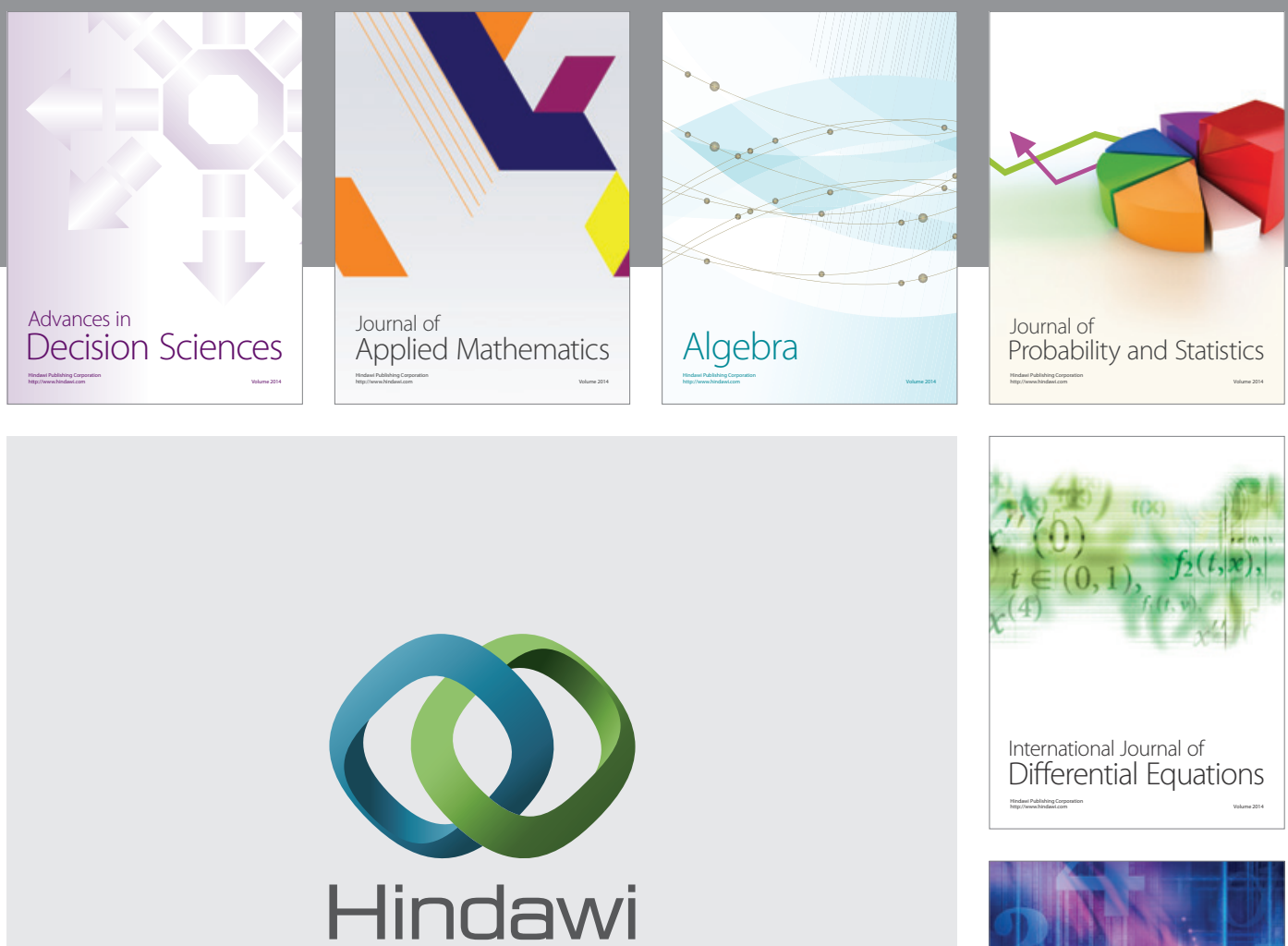

Submit your manuscripts at http://www.hindawi.com
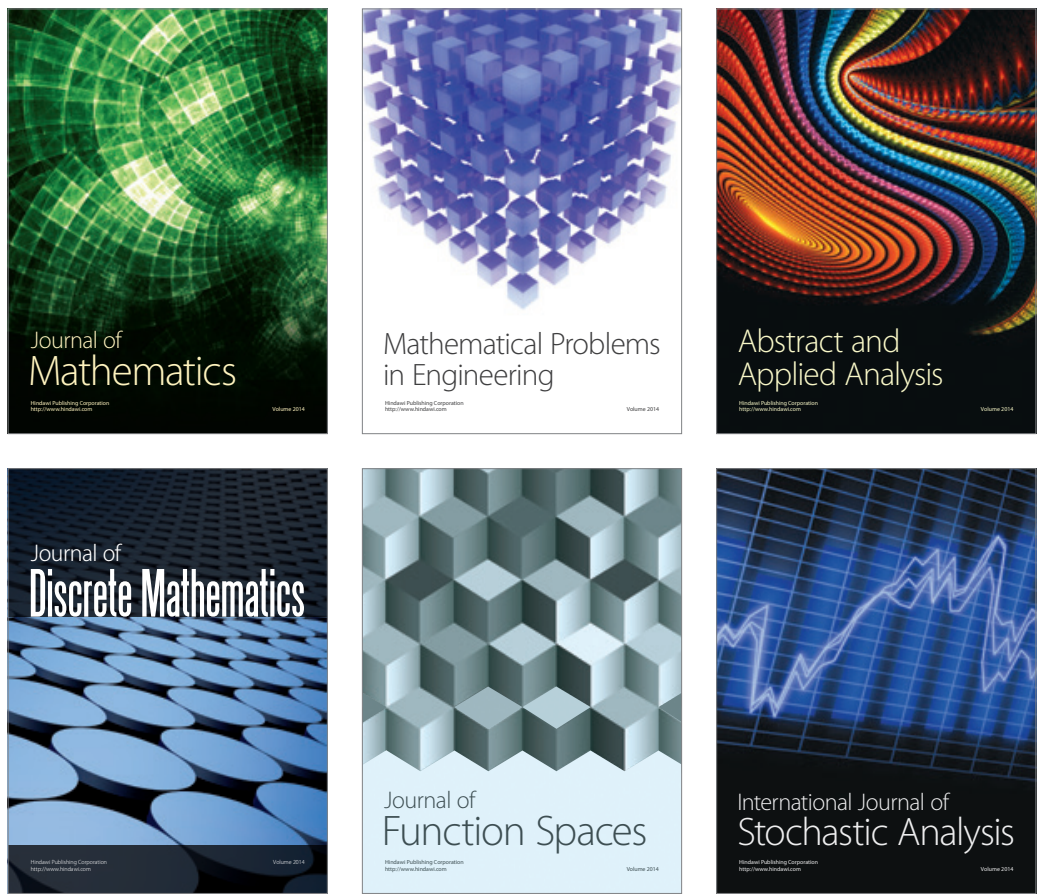

Journal of

Function Spaces

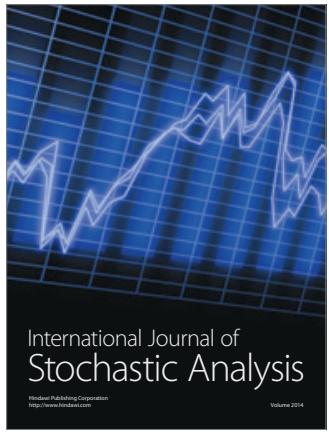

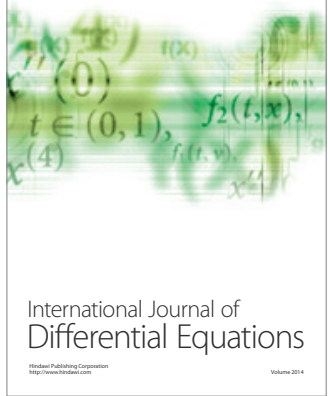
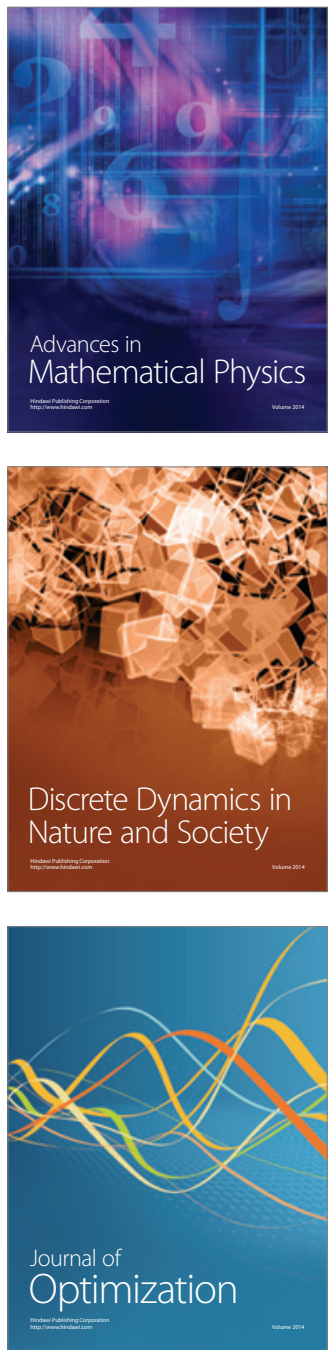